\title{
Histopathological and Immunohistochemical Diagnosis of Canine Fibrosarcoma
}

\author{
S. Subapriya*, S. Vairamuthu, N. Pazhanivel, Ravi Sundar George, \\ K. Vijayarani and M. Gokulakrishnan
}

Madras Veterinary College, Chennai, TANUVAS, India

*Corresponding author

A B S T R A C T

\section{Keywords}

Dog, cytology, fibrosarcoma, histopathology, immunohistochemistry

Article Info

Accepted:

15 May 2018

Available Online:

10 June 2018
An one year old, female, Labrador dog was presented with a history of a big mass in right hindlimb. The mass was hard on clinical examination. Fine needle aspiration cytology (FNAC) revealed pleomorphic fibrocytes with prominent nucleoli. The mass was then surgically excised. Histopathology section showed fibrocytes arranged in varying patterns with numerous mitotic figures. Immunohistochemical study showed positive expression for vimentin and no expression for cytokeratin. Based on above findings the mass was confirmed as fibrosarcoma.

\section{Introduction}

Fibrosarcoma is a malignant tumour of the fibroblasts and it is of mesenchymal origin.

Although fibrosarcomas occur in all domestic species, they are most commonly seen in adult and aged cats and dogs with mean age of 9 years (Goldschmidt and Hendrick, 2002).

This case was impressive by the external appearance of huge size of the mass.

Hence the present study was undertaken to understand the pathology of fibrosarcoma in a Labrador dog.
Fibrosarcoma is a common neoplasm that occurs in dogs, cats and other domestic animals (Kass et al., 1993; Goldschmidt and Hendrick 2002).

Sargan et al., (2005) stated that these tumours are associated with defects or rearrangements in chromosomes 11 and 30.

Genetic susceptibility (Cullen et al., 2002), various chemical substances (Smyth et al., 2001; Cullen et al., 2002), subcutaneous vaccine injections (Kass et al., 1993), chronic inflammations (Doddy et al., 1996) and traumas (Schumacher et al., 1983) have been reported as causes of fibrosarcomas in dogs 
and other animal species.

\section{Materials and Methods}

An one year old, female, Labrador dog was brought to Small Animal Clinic - Out PatientSurgery unit of Madras Veterinary College Teaching Hospital with a history of progressively increasing mass in the right hindlimb. The mass was conspicuously big, to be witnessed even at distance as a huge spherical mass. Fine needle aspiration cytology (FNAC) was performed. Radiographic investigation revealed no metastatic foci. Following this, surgery was done to excise the mass. Excised mass was collected in 10 per cent neutral buffered formalin for histopathological examination.

Cytology smear was air dried and stained with Leishman and Giemsa cocktail stain as described by Garbyal et al., (2006). In histopathological processing, paraffin embedded tissue sections were cut to 4-6 microns thickness and stained with Hematoxylin and Eosin (H\&E). For immunohistochemistry, sections were stained for Cytokeratin and Vimentin as per the procedures given by the immunnohistochemistry kit manual.

\section{Results and Discussion}

Grossly, the mass was a huge, ball like, diffuse swelling in the right hind limb, hip region, measuring about more than $15 \mathrm{~cm}$ in diameter (Fig-1). It was found extending upto the tail laterally and even seen raised dorsally above the sacrococcygeal vertebral column. It was hard, non-ulcerated with neat margins. The excised mass weighed around $3 \mathrm{kgs}$. Cytological examination revealed many pleomorphic, spindle shaped to plumpy fibrocytes (Fig-2) with multiple nucleoli. Higher magnification revealed binucleated cells, plumpy prominent nuclei with eosinophilic cytoplasm, multiple nucleoli (Fig-3) and coarse chromatin. Mitotic figures were also seen.

Histopathological examination revealed interlacing bundles of neoplastic fibrocytes arranged in sheets, herring bone (Fig-4) and in haphazard pattern. The neoplastic cells were fusiform shaped, with elongated, round to oval nuclei and indistinct eosinophilic cytoplasm. Numerous mitotic figures were seen.

Based on the cytological and histopathologic examination, the huge mass was diagnosed as fibrosarcoma. Immunohistochemical study was carried out to identify the mesenchymal origin of tumour cells as fibrosarcoma is a kind of mesenchymal tumour. In this direction, paraffin embedded tissue sections were stained with markers like Cytokeratin and Vimentin. In our findings, the sections showed no positivity for Cytokeratin (Fig-5), which ruled out epithelial tumours. However, diffuse and very strong expression of Vimentin (Fig-6) was observed which confirmed the mesenchymal origin of the tumour.

Thus pooling all the findings, cytological, histopathological and immuonhistochemical, the giant mass was confirmed as fibrosarcoma. In our study, the tumour was identified in a relatively young dog of just one year old. However earlier reports have reported the occurrence of this tumour in adult dogs with a mean age of 9 years (Goldschmidt and Hendrick 2002). As in our case, skin and subcutis have been reported as the primary sites of occurrence of fibrosarcomas in dogs and other domestic animals by Goldschmidt and Hendrick (2002). However Avci et al., (2012) has reported a rare case of intestinal fibrosarcoma in a 11 year old Cocker Spaniel dog. 


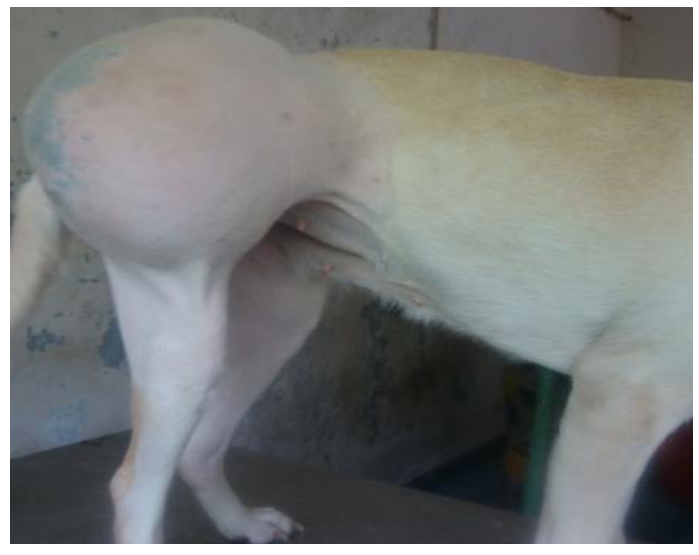

Fig-1 Dog-Fibrosarcoma - Right thigh-

Huge, spherical mass

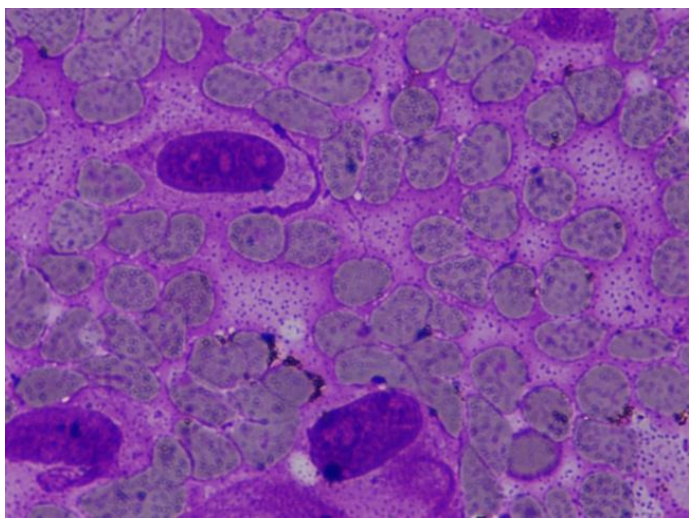

Fig-3 Dog - Fibrosarcoma- FNAC

Prominent,multiple nucleoli-LG-100x

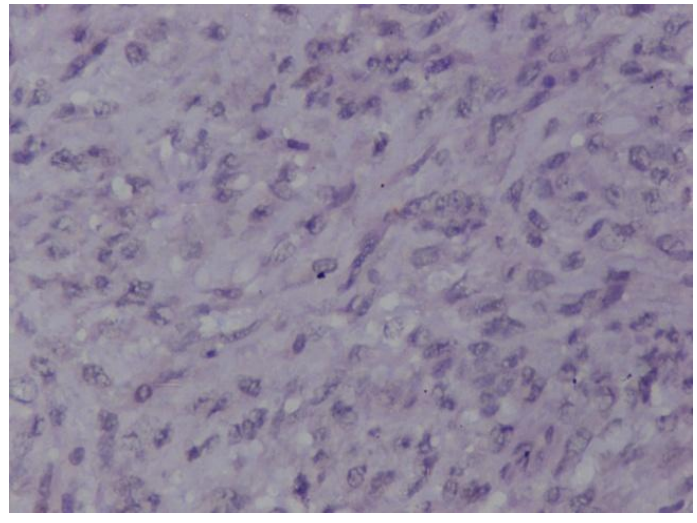

Fig-5 Dog - Fibrosarcoma - Immunohisto

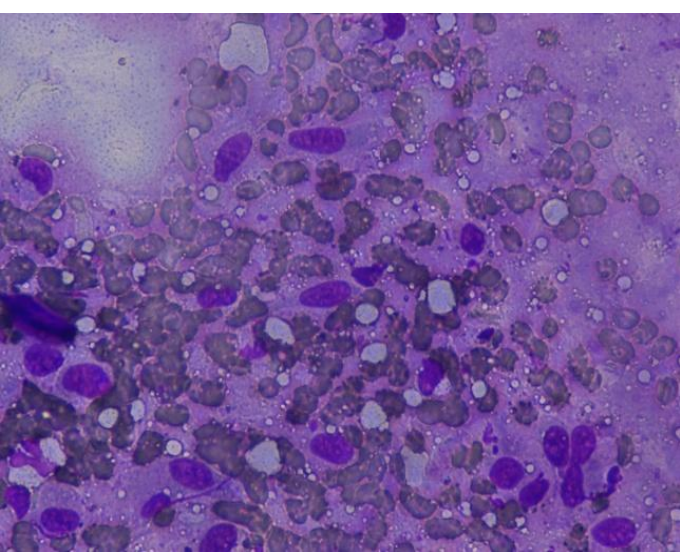

Fig-2 Dog - Fibrosarcoma- FNAC

Spindle to plump cells- LG - 40x

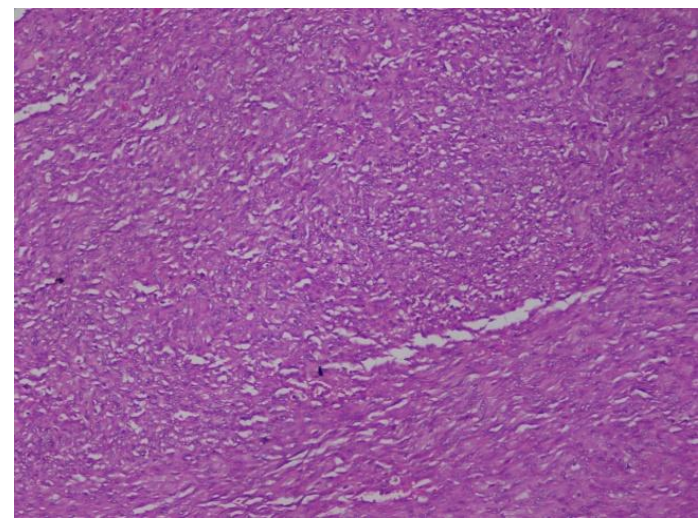

Fig-4 Dog -Fibrosarcoma-Neoplastic cells arranged in herring bone pattern- H\&E- 10x

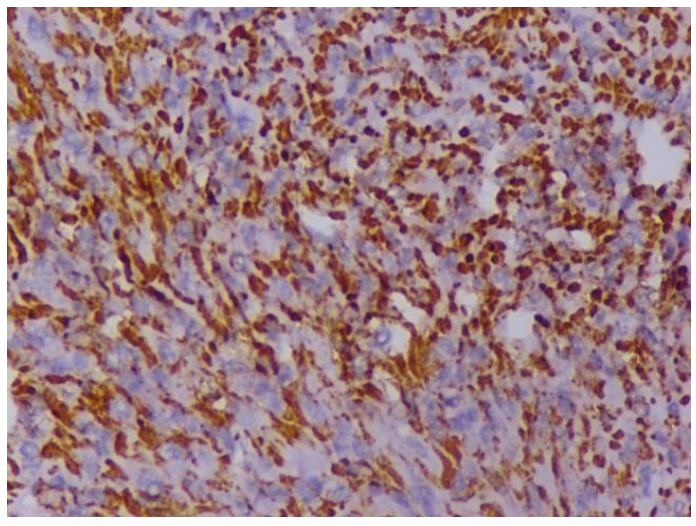

Fig-6 Dog - Fibrosarcoma - Immunohisto

Chemistry-Vimentin-Diffuse and strong positive expression-40x 
The numerous mitotic figures seen in microscopic examination were highly suggestive of the malignant nature of the tumour. In concordance with this, the animal had a fatal end during the follow up for tumour free survival time.

\section{Acknowledgement}

The authors are thankful to the Dean, Madras Veterinary College, TANUVAS, for providing the necessary facilities to carry out the work.

\section{References}

Avci, H., R. Yaygingul, M. Gultekin, E.T. Epikmen, K. Ural, A. Belge and Birincioglu. S.S. 2012. Primary intestinal fibrosarcoma caused by intestinal perforation in a dog: a case report. Veterinarni Medicina, 57 (6): 314-319

Cullen, J.M., R. Page and Misdorp, W. 2002. An overview of cancer pathogenesis, diagnosis, and management. In: Meuten DJ (ed.): Tumors in Domestic Animals. 4th ed. Iowa State Press, Iowa. 3-44.

Doddy, F.D., L.T. Glickman, N.W. Glickman and Janovitz, E.B. 1996: Feline fibrosarcomas at vaccination sites and non-vaccination sites. Journal of Comparative Pathology. 114: 165-174.

Gallati, W.W., 1956. Fibrosarcoma associated with the cysticercus of Taenia taeniaeformis in the liver of a muskrat. Ohio Journal of Science 56: 71-75.

Garbyal, R.S., N.Agarwal and Kumar, P. 2006. Leishman-Giemsa Cocktail, An Effective Romanowsky stain for air dried cytologic smears. Acta Cytol, 50: 403-406.

Goldschmidt, M.H., and Hendrick M.J. 2002. Tumors of the skin and soft tissues. In: Meuten DJ (ed.): Tumors in Domestic Animals. 4th ed. Iowa State University Press, Iowa. 45-117.

Kass, P.H., W.G. Barnes, W.L. Splanger, B.B. Chomel and Culbertson, M.R. 1993): Epidemiological evidence for a Veterinarni Medicina, 57, 2012 6: 314319 Case Report 319 casual relation between vaccination and fibrosarcoma tumorigenesis in cats. Journal of the American Animal Hospital Association 203, 396-405.

Sargan, D.R., B.S. Milne, J.A. Hernandez, P.C. O'Brien, M.A. Ferguson-Smith, T. Hoather and Dobson, J.M. 2005. Chromosome rearrangements in canine fibrosarcomas. J Hered 96(7): 766-773

Schumacher, J., J.P. Watkins, S.R. Wilson and Foreman, M.E. 1983: Burn-induced neoplasia in two horses. Equine Veterinary Journal. 18: 410-412.

Smyth, M.J., N.Y. Crowe and Godfrey, D.I. 2001. NK cells and NKT cells collaborate in host protection from methylcholanthrene-induced

fibrosarcoma.

International

\section{How to cite this article:}

Subapriya S., S. Vairamuthu, N. Pazhanivel, Ravi Sundar George, K. Vijayarani and Gokulakrishnan M. 2018. Histopathological and Immunohistochemical Diagnosis of Canine Fibrosarcoma. Int.J.Curr.Microbiol.App.Sci. 7(06): 1376-1379.

doi: https://doi.org/10.20546/ijcmas.2018.706.162 